\title{
Role of renin-angiotensin system antagonists on long-term mortality post-percutaneous coronary intervention in reduced and preserved ejection fraction
}

\author{
Hamish C. Prosser ${ }^{1,2} \cdot K$ ah Yong Peck ${ }^{1,2} \cdot$ Diem Dinh $^{3} \cdot$ Louise Roberts ${ }^{1,2} \cdot$ Jaya Chandrasekhar ${ }^{1,2} \cdot$ Angela Brennan $^{3}$. \\ Stephen J. Duffy ${ }^{3,4}$. David Clark ${ }^{5}$. Andrew E. Ajani ${ }^{6}$. Ernesto Oqueli ${ }^{7,10} \cdot$ Martin Sebastian $^{8} \cdot$ Christopher M. Reid $^{9,3}$. \\ Melanie Freeman ${ }^{1} \cdot$ Jithin K. Sajeev ${ }^{1,2} \cdot$ Andrew W. Teh ${ }^{1,2,5}$
}

Received: 29 August 2021 / Accepted: 21 December 2021 / Published online: 20 January 2022

(c) The Author(s) 2022

\begin{abstract}
Aims The use of angiotensin-converting enzyme inhibitors (ACEi) or angiotensin II-receptor blockers (ARBs) post-myocardial infarction (MI) is supported by evidence based on trials performed in the thrombolysis era. This was prior to primary percutaneous coronary intervention (PCI) being routine practice, and with little direct evidence for the use of these medications in patients with preserved left ventricular (LV) function. This study sought to determine whether there is an association between ACEi/ARB use after PCI for acute coronary syndrome (ACS) and long-term all-cause mortality, with a particular focus on patients with preserved LV function.

Methods This multicentre, observational study evaluated prospectively collected data of 21,388 patients ( $>18$ years old) that underwent PCI for NSTEMI and STEMI between 2005 and 2018, and were alive at 30 day follow-up.

Results In total, $83.8 \%$ of patients were using ACEi/ARBs. Kaplan-Meier analysis demonstrated ACEi/ARB use was associated with a significantly lower mortality in the entire cohort (15.0 vs. $22.7 \% ; p<0.001)$ with a mean follow-up of 5.58 years; and independently associated with $24 \%$ lower mortality by Cox proportional hazards modelling (HR 0.76, CI $0.67-0.85$, $p<0.001)$. ACEi/ARB therapy was also associated with significantly lower mortality in patients with reduced or preserved LV function, with greater survival benefit with worse LV dysfunction.

Conclusion ACEi/ARB therapy post-PCI is associated with significantly lower long-term mortality in patients with reduced and preserved LV function. These findings provide contemporary evidence for using these agents in the current era of routine primary PCI, including those with preserved EF.
\end{abstract}

Andrew W. Teh

andrew.teh@easternhealth.org.au

1 Department of Cardiology, Eastern Health, Box Hill Hospital, Level 2, 8 Arnold Street, Box Hill, VIC 3128, Australia

2 Eastern Health Clinical School, Monash University, Melbourne, VIC, Australia

3 Department of Epidemiology and Preventive Medicine, Monash University, Melbourne, VIC, Australia

4 Department of Cardiology, Alfred Health, The Alfred Hospital, Melbourne, VIC, Australia
5 Department of Cardiology, Austin Hospital Clinical School, The University of Melbourne, Melbourne, VIC, Australia

6 Department of Cardiology, Royal Melbourne Hospital, Parkville, VIC, Australia

7 Department of Cardiology, Ballarat Health Services, Ballarat, VIC, Australia

8 Department of Cardiology, Barwon Health, University Hospital, Geelong, VIC, Australia

9 School of Public Health, Curtin University, Perth, WA, Australia

10 School of Medicine, Deakin University, Geelong, Australia 


\section{Graphical abstract}

\section{ACEi/ARB therapy post PCI for NSTEMI/STEMI is associated with lower long-term mortality}

\section{1,388 patients post PCI for NSTEMI and STEMI}

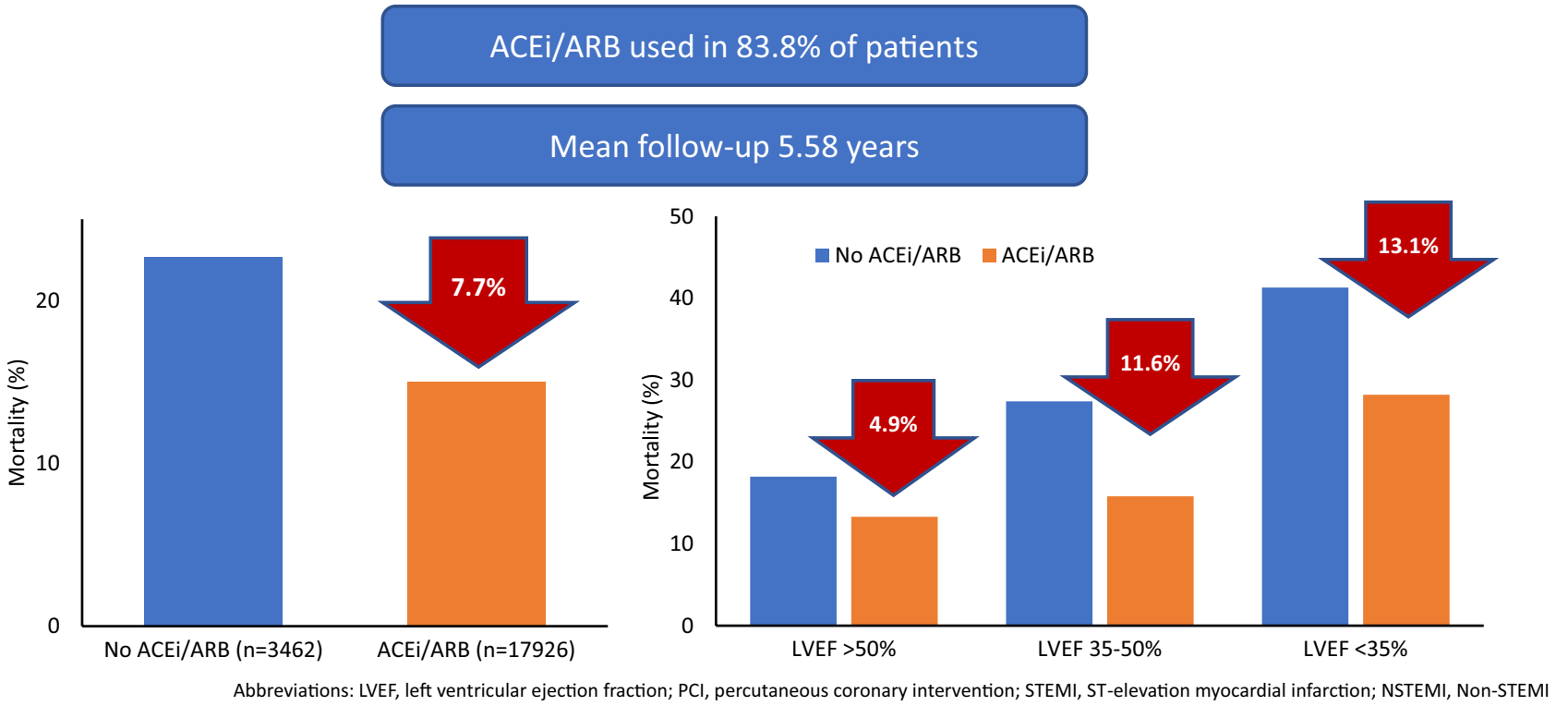

Keywords Angiotensin converting enzyme inhibitors · Angiotensin receptor blockers · STEMI/NSTEMI · Percutaneous coronary intervention $\cdot$ Heart failure

\section{Introduction}

Optimal medical therapy plays a critical role in preventing further cardiovascular events and improving clinical outcomes following percutaneous coronary intervention (PCI) for ACS [1-3]. As such, international guidelines recommend multiple medications for secondary-prevention including anti-platelet agents, a $\beta$-adrenergic receptor blocker ( $\beta$-blocker), and a statin [4-7]. Angiotensin-converting enzyme inhibitors (ACEi) or angiotensin receptor blockers (ARBs) are also recommended as first-line therapy in patients with concomitant heart failure (HF), left ventricular (LV) dysfunction (ejection fraction (EF) $\leq 40 \%$ ), anterior MI, diabetes or hypertension, and in those with stable chronic kidney disease (CKD) [4-8]. Guidelines also recommend that ACEi may be considered for all patients regardless of these associated factors, but this is supported by a reduced level of evidence (Class IIa, level A-B evidence) [4-8].

The evidence underlying guideline recommendations comes from large trials mostly performed over 20 years ago, during an era prior to routine and/or primary PCI, where thrombolysis was often performed, with greater subsequent mortality compared to the present time. The last 20 years have seen major changes in the management of ACS, resulting in a greater proportion of patients with preserved LV function, primarily due to increased use of primary PCI, and a routine early invasive strategy in NSTEMI $[2,9]$.

The aims of this study were (1) identify prescribing practices of RAS inhibitors (ACEi and ARBs) in patients post-PCI for MI between 2005 and 2018; (2) evaluate the long-term survival benefits of ACEi/ARBs in an unselected cohort of patients that underwent PCI for STEMI or NSTEMI; (3) determine the effects of ACEi/ARB therapy in patients with reduced and preserved LV function by subgroup analysis.

\section{Methods}

\section{Study design and patient population}

This multicenter, observational study utilized prospectively collected data from the Melbourne Interventional Group (MIG) registry, an Australian registry that collects comprehensive data on consecutive patients undergoing PCI from 
six major public (government funded) hospitals within Victoria, Australia [10]. Data from a total of 21,388 patients that underwent PCI for STEMI and NSTEMI between January 2005 and September 2018 were evaluated. Inclusion criteria required the patient to be $>18$ years old, undergo PCI for NSTEMI or STEMI, and be contactable at 30-day follow up where their use of ACEi/ARB and clinical outcomes were recorded. Exclusion criteria included patients that died prior to, or were not contactable at 30-day follow up, and those in which use of ACEi/ARB was not able to be determined (discharge medications are not recorded in the MIG registry). Long-term mortality was identified via linkage to the Australian National Death Index, with multiple patient demographics cross-matched with the MIG registry to identify deceased patients. The ethics committee of each participating hospital had approved registry participation. Consent was obtained in all participants via an "opt-out" model. This study abided by the ethical guidelines of the 1975 Declaration of Helsinki.

\section{Outcomes}

The primary outcome of this study was long-term, all-cause mortality. The cohort was stratified by patient LV ejection fraction (EF) into $<35 \%, 35-50 \%$ and $>50 \%$. LVEF was measured or estimated within 4 weeks of patient admission, the vast majority during the inpatient stay.

\section{Statistical analysis}

Continuous variables are expressed as mean \pm standard deviation, with categorical variables expressed as numbers and percentages as indicated. Differences between groups in discrete variables relating to patient characteristics and clinical outcomes were analyzed by Pearson Chi-squared tests. All data were tested for normal distribution prior to selecting the appropriate statistical test. Where appropriate, independent sample $t$ tests (for normally distributed data) or Kruskal-Wallis equality-of-populations rank tests (for nonnormally distributed data) were used for continuous variables. Survival curves were determined by Kaplan-Meier analysis, with differences in survival assessed by log-rank tests. A $p$ value of $<0.05$ was considered to indicate statistical significance. Differences in baseline characteristics which may have acted as potential confounders to the analysis were accounted for using Cox proportional multivariable modelling, as previously published [11]. Variables found to have a $p$ value $<0.10$ in the univariable model were considered for inclusion in the multivariable model to identify whether they remained statistically significant and independent predictors of mortality. The full list of variables included in this modelling is included in Supplementary Table 1.
Statistical analyses were performed using Stata software (Stata 16.0, College Station, TX, USA).

\section{Results}

\section{Baseline patient characteristics}

Between 2005 and 2018 23,942 consecutive patients underwent PCI for STEMI or NSTEMI. Of these 2554 were excluded and the remaining 21,388 patients alive at 30 day follow-up were evaluated (Fig. 1). At 30 days, 17,926 patients (83.8\%) were taking an ACEi/ARB, while 3462 (16.2\%) were not (Table 1). At baseline, those who were on ACEi/ARB therapy at 30 days were younger, hypertensive, with elevated BMI, and a family history of CAD. The group not prescribed ACEi/ARB had significantly more women and comorbidities including chronic lung disease, prior MI, prior CABG, prior HF, prior valvular surgery, peripheral vascular disease, cerebrovascular disease, renal disease requiring dialysis, and rheumatoid arthritis (Table 1).

These baseline differences between treatment groups from the entire cohort were generally mirrored in the subgroups EF $35-50 \%(N=8221)$ and $\mathrm{EF}>50 \%(N=12,091)$. Few patient characteristics differed between treatment groups in the smaller cohort of patients with $\mathrm{EF}<35 \%(N=1076)$, however, significantly fewer were on ACEi/ARB if hypertensive or a current smoker, and a greater proportion were on ACEi/ARB in the setting of prior PCI.

\section{Prescribing practices of ACEi/ARBs at 30-day follow-up}

Observing the use of ACEi/ARBs in all patients post-PCI between 2005 and 2018, the proportion of patients receiving ACEi/ARB each year fluctuated mildly between 80 and $87 \%$ with a small but statistically significant reduction in ACEi/ ARB use over the study period $(p=0.001)$, and remained significantly greater than patients not using ACEi/ARB ( $p<0.001$; Fig. 2). Of the $83.8 \%$ of patients prescribed an ACEi/ARB in this study, $82.8 \%$ were prescribed ACEi and $18.2 \%$ an ARB (Table 2).

There were some statistically significant differences in medication regimens identified between those that were and were not on ACEi/ARB therapy (Table 2). Patients on ACEi/ ARB had a significantly higher proportion also on aspirin, $\mathrm{P} 2 \mathrm{Y} 12$ inhibitors, warfarin, eplerenone, $\beta$-blocker and statin therapy. Those not prescribed ACEi/ARB were more frequently prescribed calcium channel blockers, ezetimibe, non-vitamin $\mathrm{K}$ oral anticoagulants (NOAC) and nitrate therapy. The use of spironolactone did not differ between those on ACEi/ARB therapy or not. The greater prescribing of 
Fig. 1 Flow diagram illustrating the study population and subgroups. ACE $i$ angiotensin converting enzyme inhibitor, $A R B$ angiotensin receptor blocker, $E F$ left ventricular ejection fraction, NSTEMI non-ST-segment elevation myocardial infarct, STEMI ST-segment elevation myocardial infarct

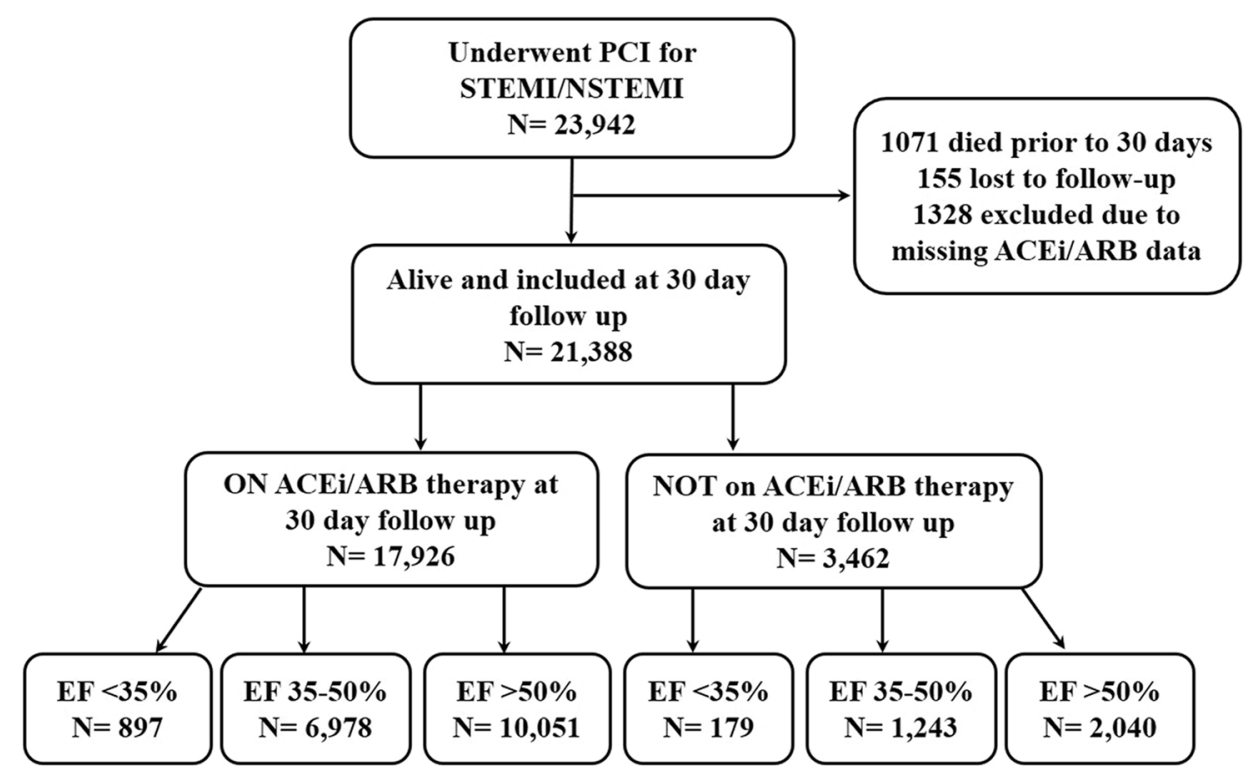

aspirin, statin, and $\beta$-blockers to those on ACEi/ARB was consistent across all LVEF subgroups (Table 2).

\section{Cardiac status and angiography/PCl characteristics}

All patients underwent PCI, with $87 \%$ of STEMI patients on ACEi/ARB therapy at 30 days compared to $80 \%$ for NSTEMI $(p<0.001$; Table 3$)$. A significantly greater proportion of patients were on ACEi/ARB therapy if they had an out of hospital cardiac arrest, underwent thrombolysis, or had a successful PCI (Table 3).

\section{Clinical outcomes}

On Kaplan-Meier analysis of the entire cohort, ACEi/ ARB therapy was found to be associated with significantly reduced mortality ( $15.0 \%$ vs. $22.7 \%, p<0.001$; mean follow up 5.58 years; Fig. 3). This significant reduction in mortality was also present across each EF subgroup, with ACEi/ARB being associated with improved survival by $4.9 \%, 11.6 \%$ and $13.1 \%$ in patients with LVEF $>50 \%, 35-50 \%$ and $<35 \%$ respectively, suggesting a trend of increased therapeutic benefit with increasing LV dysfunction (Fig. 3).

For the unselected cohort, Cox proportional hazards modelling demonstrated that ACEi and ARB use were independently associated with reduced mortality (Fig. 4, Supplementary Table 1). On subgroup analysis with patients stratified by $\mathrm{EF} \%$, ACEi/ARB remained independently associated with improved survival in those with EF 35-50 and $>50 \%$ (both $p<0.001$ ); however, did not reach significance in those with $\mathrm{EF}<35 \%$ despite a trend towards survival benefit (HR 0.69 , CI 0.45-1.1, $p=0.088$; Supplementary Table 1). A full table of all variables analyzed by Cox proportional hazards modelling can be found in Supplementary Table 1.

\section{Discussion}

The key findings of this study were that (1) the use of ACEi/ ARB post PCI for NSTEMI/STEMI is high (83.8\%); (2) ACEi/ARB therapy in patients post-PCI for NSTEMI or STEMI is associated with a significant long-term survival benefit; (3) this survival benefit is evident in patients with both preserved and reduced LV function.

Current international guidelines recommend the use of ACEi (or ARBs when ACEi are not tolerated) for patients with STEMI and NSTEMI with concomitant heart failure, LAD lesion, diabetes, or hypertension, and in those with stable CKD after NSTEMI with Class IA evidence [4-8]. Lower levels of evidence support their use outside of these criteria [4-8]. The current study found $83.8 \%$ of patients were prescribed ACEi/ARB therapy at 30 days post PCI. Given that $60.8 \%$ had hypertension, $43.9 \%$ had an $\mathrm{EF}<50 \%$, $21.1 \%$ were diabetic, $20.4 \%$ had an eGFR $<60$, and $4.2 \%$ had reported heart failure at baseline prior to PCI; while $35.5 \%$ of presentations involved the left anterior descending or left main coronary arteries, prescribing practices appear to follow current guidelines. Use of ACEi/ARB also appeared to be associated with the severity of the presentation; with ACEi/ARB use significantly greater in those who had a STEMI compared to those with a NSTEMI or had an out-of-hospital cardiac arrest. The greater proportion of patients on ACEi/ARB with STEMI compared to NSTEMI is consistent with the greater level of evidence recommending their use in patients with STEMI (regardless 


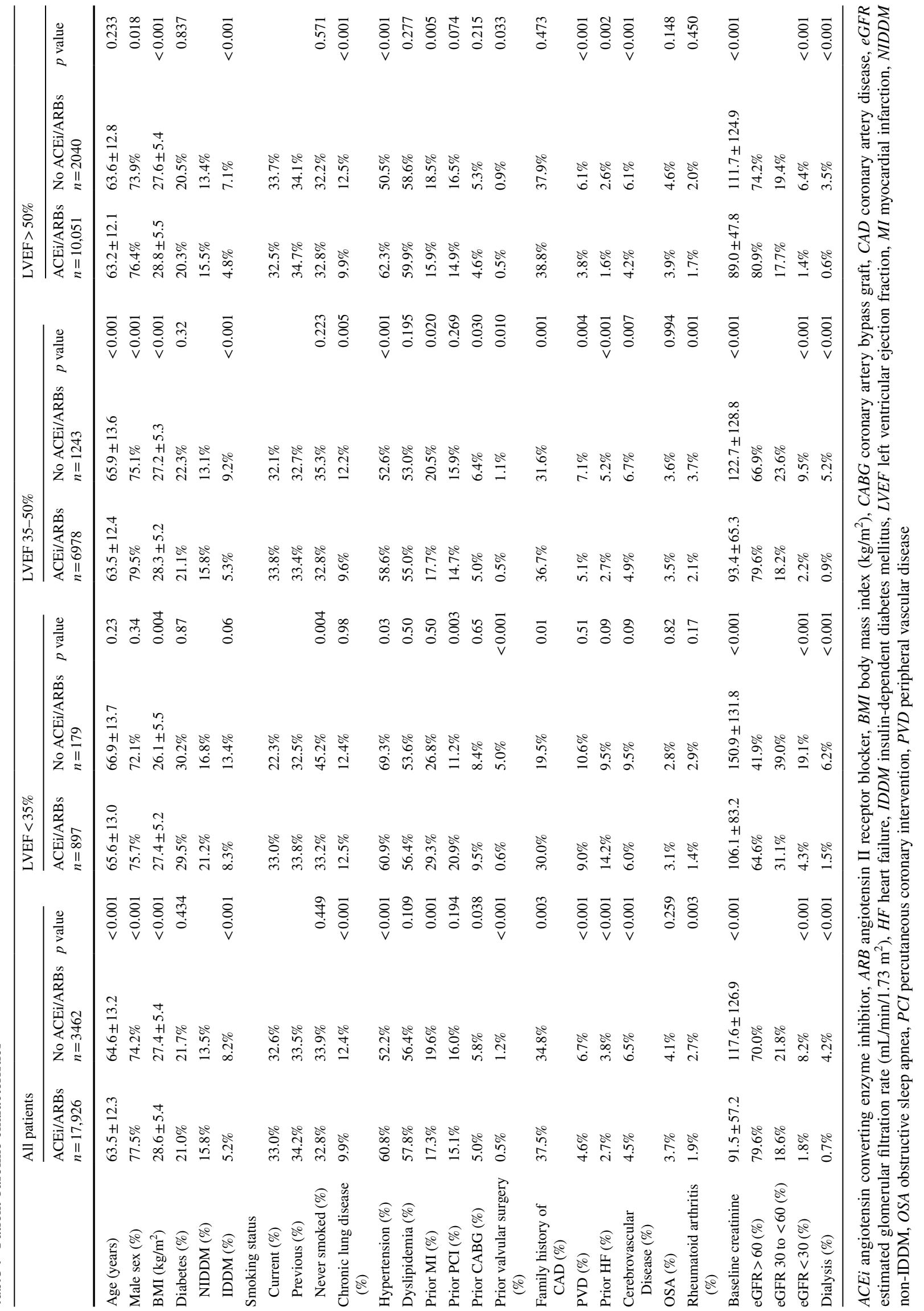


Fig. 2 ACEi/ARB use across the study period 2005-2018. The total rate of ACEi/ARB use is $83.8 \%(p<0.001$ versus no ACEi/ARB use). The trend over time shows a small but significant decline in ACEi/ARB use $(p=0.001) . A C E i$ angiotensin converting enzyme inhibitor, $A R B$ angiotensin receptor blocker

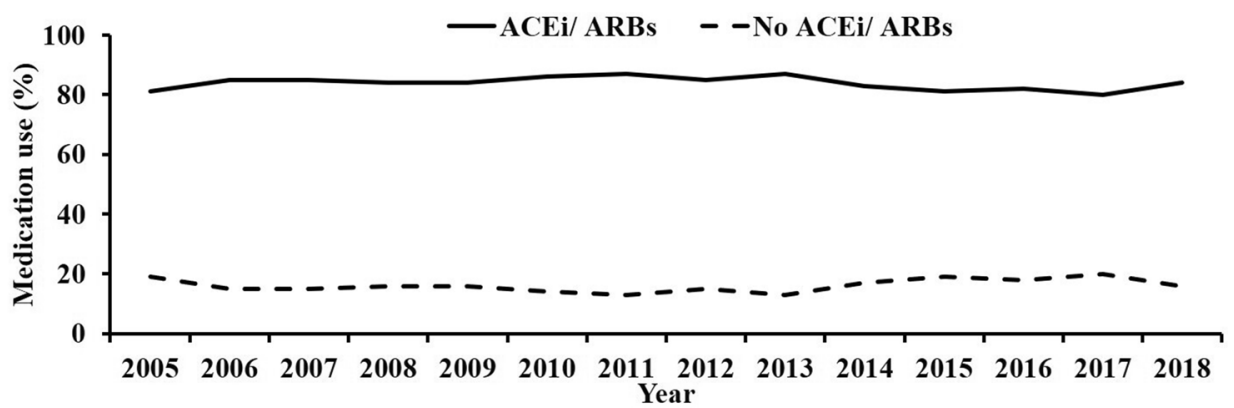

of LV function) as per ACC/AHA guidelines. Notably, this evidence was obtained during the pre-PCI era [4, 8]. Recent evidence supports this prescribing practice in which RAS inhibition provided greater survival benefit in 6,762 pairs of unselected and propensity score-matched Korean patients with STEMI vs. NSTEMI patients 2-years post-PCI in an observational study [12].

The present study found that ACEi/ARB therapy was associated with significantly reduced long-term all-cause mortality in patients post-PCI for MI by Kaplan-Meier and Cox proportional hazards analyses of the entire cohort. This is consistent with original reports undertaken prior to PCI becoming standard practice. More recent smaller cohort studies have provided data in the current PCI era, where ACEi/ARB therapy was associated with significantly reduced 4-year all-cause mortality in 813 unselected and propensity-matched pairs of patients post-PCI for NSTEMI in Spain [13]; and was associated with significantly improved 5-year survival in 5563 unselected and propensitymatched Japanese patients presenting with MI (with $>80 \%$ of patients treated with PCI) [14].

There is currently less direct evidence supporting ACEi/ ARB in patients with preserved LV function following PCI for a MI. Thus, the current study stratified patients by $\mathrm{EF} \%(\mathrm{EF}<35 \%, 35-50 \%$ and $>50 \%)$ and revealed that ACEi/ARB therapy was associated with significantly higher survival across all subgroups irrespective of LVEF on Kaplan-Meier analysis. This provides important contemporary evidence to support ACEi/ARB therapy use in all patients post-PCI for STEMI/NSTEMI, regardless of LV function, with particular relevance to the modern era of early revascularization with primary PCI. Cox proportional hazards modelling demonstrated that ACEi/ARB therapy was independently associated with improved survival for those with EF 35-50 and $>50 \%$, but despite a clear trend, did not reach significance in those with $\mathrm{EF}<35 \%$, likely attributable to the smaller sample size in this subgroup.

Analysis of ACEi and ARB therapy by Cox proportional modelling revealed each to be associated with a statistically significant survival benefit for the entire unselected cohort. Strong evidence exists for ACEi use in reducing mortality in LV dysfunction post MI in the pre-PCI era [15-17], while evidence for the use of ACEi in those with preserved LV function post MI has been an area of debate. HOPE and EUROPA placebo-controlled trials in patients with stable CAD and without heart failure reported ACEi to significantly reduce mortality; however only $52 \%$ and $64 \%$ of patients had a prior MI, and just $18 \%$ and $55 \%$ had undergone revascularization in each trial, respectively $[18,19]$. In contrast, the placebo-controlled PEACE trial found the ACEi Trandolapril to have no significant survival benefit in patients with stable CAD without heart failure, but again contained low rates of prior MI (55\%) and PCI (42\%) [20]. Trials focused on investigating the effect of ACEi/ARB in secondary prevention with preserved LV function are scarce, with one placebo-controlled study of 406 Japanese patients with a history of coronary intervention and preserved LVEF reporting Candesartan significantly reduced cardiovascular death (RR 0.47; 95\% CI 0.24-0.93, $p=0.03$ ) [21]. In contrast, a more recent study of 988 patients with STEMI and preserved EF that underwent PCI showed no longterm survival benefit of ACEi/ARB therapy after a median of 4.6 years (HR 0.86, CI 0.56-1.33, $p=0.50$ ) [22]. The reasons why that study conflicts with the current findings may be due to the much smaller sample size, and exclusion of patients with eGFR $<60 \mathrm{~mL} / \mathrm{min} / 1.73 \mathrm{~m}^{2}$ or NSTEMI. Furthermore, differences in study period (2002-2011 vs. 2005-2018) may also account for the variation in results due to changes in PCI practices. Recent prospective cohort and observational studies report ACEi and ARB therapy were each associated with significant and comparable survival benefits in patients presenting with STEMI and NSTEMI that underwent PCI in patients with preserved LV function $[23,24]$. Taken together, both the current and recent relevant studies support the use of ARBs/ACEi in patients post PCI with preserved EF.

The efficacy of ACEi-ARB in modifying outcomes after PCI rely upon tolerance to these medications. The most common causes of intolerance include drug reactions, hypotension or hyperkalaemia often associated with renal impairment. Consistent with this, in the current study, those not-receiving ACEi/ARB therapy had a significantly reduced eGFR compared to those receiving ACEi/ARB therapy. Having a reduced renal function may potentially carry a worse 


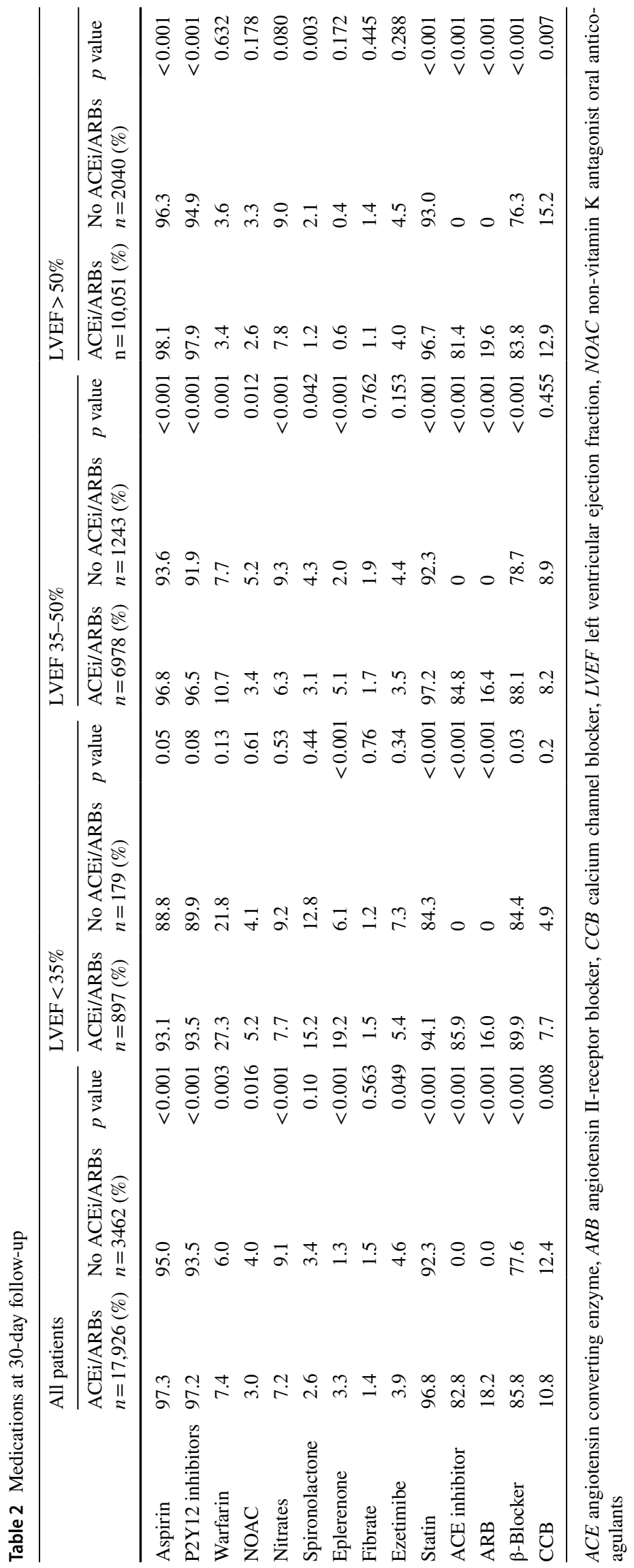




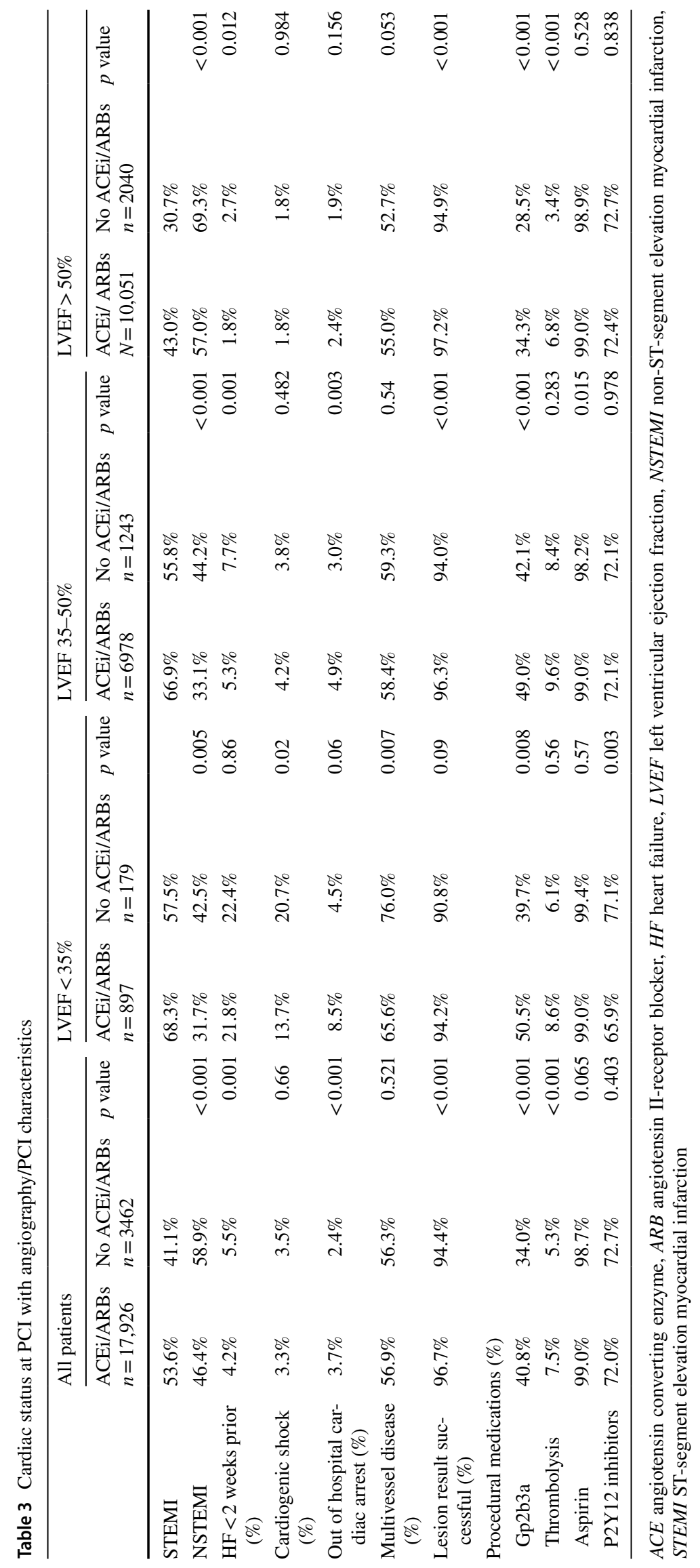


Fig. 3 Kaplan-Meier survival analysis for all-cause mortality stratified by left ventricular ejection fraction. Within each group, ACEi/ARB therapy was associated with significantly lower mortality compared to no ACEi/ARB therapy (all $p<0.001)$. ACEi angiotensin converting enzyme inhibitor, $A R B$ angiotensin receptor blocker, $E F$ ejection fraction

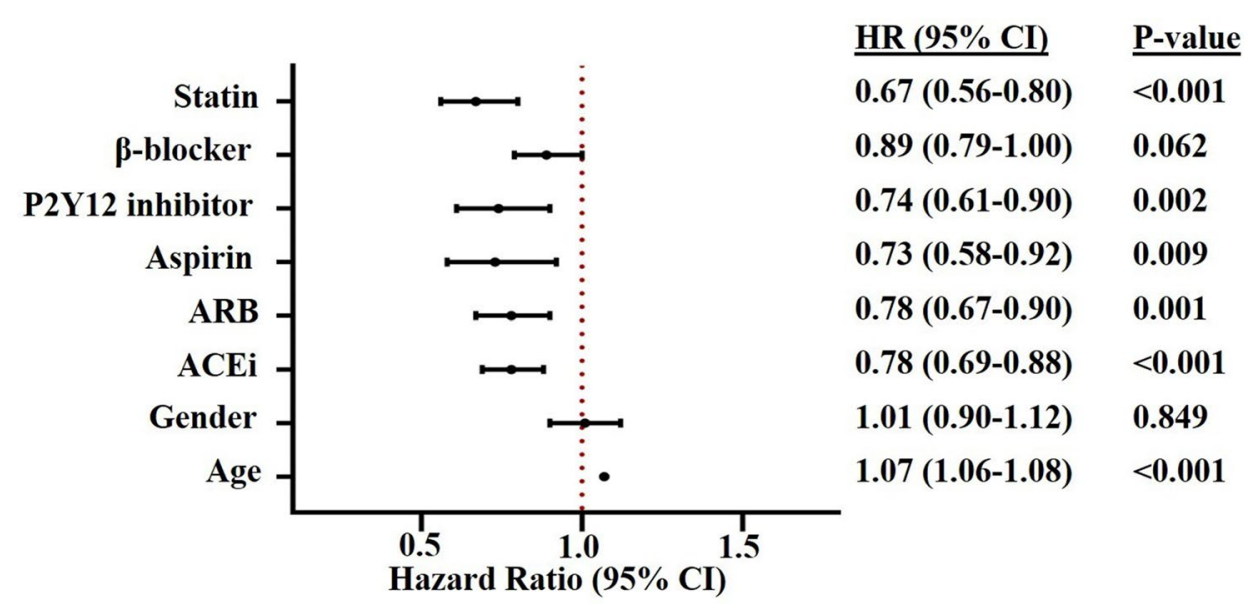

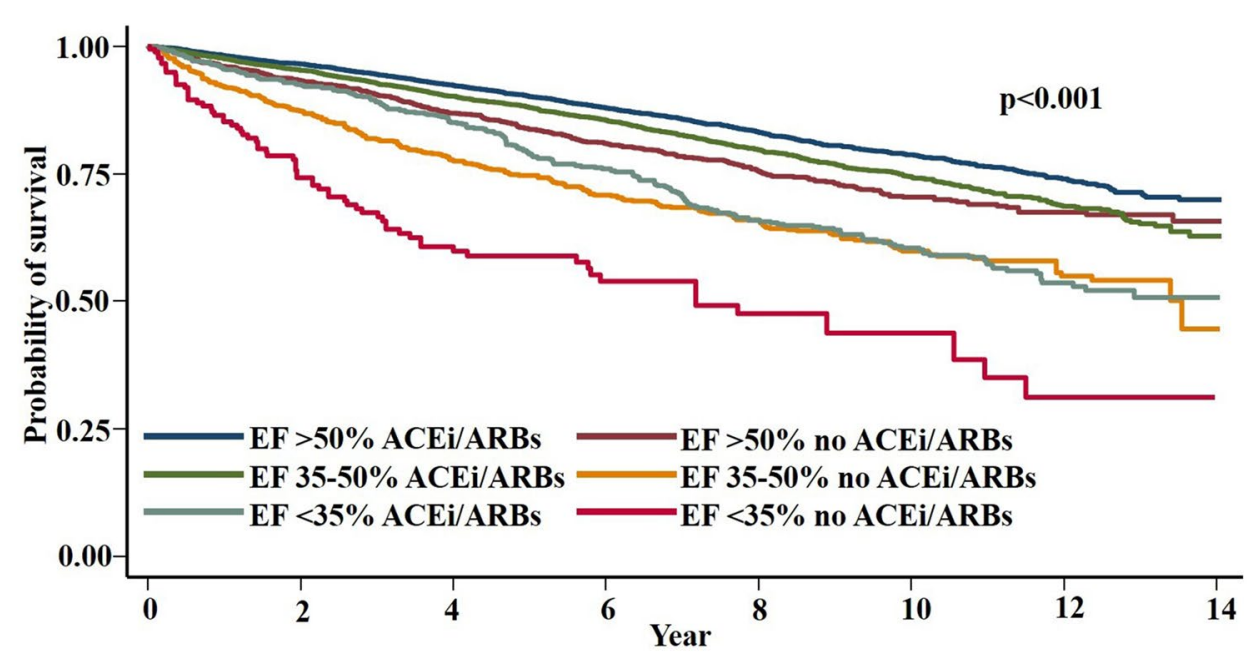

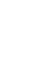

.

Fig. 4 Cox proportional hazards survival analysis for all-cause mortality of the entire cohort $(N=21,388)$. Value of $<1.0$ indicates improved survival. $A C E i$ angiotensin converting enzyme inhibitor, $A R B$ angiotensin receptor blocker 
cessation prior to this time-point not collected within our dataset. Unexpectedly, several medications used for secondary prevention after ACS were disproportionally used in the ACEi/ARB group as described above. The dose, specific drug type, long-term adherence or discontinuation of $\mathrm{ACEi} / \mathrm{ARB}$ and/or other medications after 30 days were not recorded, although the latter would potentially diminish the associated survival benefits with ACEi/ARB. With the focus of this study on long-term survival outcomes, only patients that were alive and contactable at 30 days were included, thus any potential clinical effects of ACEi/ ARB therapy prior to 30 days of PCI were not assessed, nor were major adverse cardiovascular events, change in LVEF, or other parameters of patient health recorded as part of this study. Lastly, routine use of Angiotensin Reception Neprilysin Inhibitors (ARNIs) in Australia was only commenced in the final year of our dataset and was therefore not included.

\section{Conclusions}

This large observational study found ACEi/ARB therapy was associated with significant long-term survival benefit in patient's post-PCI for STEMI/NSTEMI. This survival benefit is apparent in patients with both preserved and reduced LV function. These findings provide contemporary evidence to support the use of these agents in patients treated with PCI for STEMI/NSTEMI, irrespective of their baseline left ventricular function.

Supplementary Information The online version contains supplementary material available at https://doi.org/10.1007/s00392-021-01985-x.

Author contributions HP and AT: conceptualization, methodology, data curation, writing - original draft preparation, visualization. DD and AB: formal analysis. LR, JC, SD, DC, AA, EO, MS, CR, MF, JS, AT: investigation, writing - review and editing.

Funding Open Access funding enabled and organized by CAUL and its Member Institutions. Professor Duffy's work is supported by a National Health and Medical Research Council of Australia (Grant number 1111170). Professor Reid is supported by a NHMRC Principal Research Fellowship (reference no. 1136372). The authors have no financial relationships with industry or other entities connected with this study.

Open Access This article is licensed under a Creative Commons Attribution 4.0 International License, which permits use, sharing, adaptation, distribution and reproduction in any medium or format, as long as you give appropriate credit to the original author(s) and the source, provide a link to the Creative Commons licence, and indicate if changes were made. The images or other third party material in this article are included in the article's Creative Commons licence, unless indicated otherwise in a credit line to the material. If material is not included in the article's Creative Commons licence and your intended use is not permitted by statutory regulation or exceeds the permitted use, you will need to obtain permission directly from the copyright holder. To view a copy of this licence, visit http://creativecommons.org/licenses/by/4.0/.

\section{References}

1. Iqbal J, Zhang YJ, Holmes DR et al (2015) Optimal medical therapy improves clinical outcomes in patients undergoing revascularization with percutaneous coronary intervention or coronary artery bypass grafting: insights from the Synergy Between Percutaneous Coronary Intervention with TAXUS and Cardiac Surgery (SYNTAX) trial at the 5-year follow-up. Circulation 131(14):1269-1277

2. Szummer K, Wallentin L, Lindhagen L et al (2017) Improved outcomes in patients with ST-elevation myocardial infarction during the last 20 years are related to implementation of evidence-based treatments: experiences from the SWEDEHEART registry 1995-2014. Eur Heart J 38(41):3056-3065

3. Yudi MB, Farouque O, Andrianopoulos N et al (2020) Prognostic Significance of Suboptimal Secondary Prevention Pharmacotherapy After Acute Coronary Syndromes. Intern Med J. https://doi.org/10.1111/imj.14750

4. O'Gara PT, Kushner FG, Ascheim DD et al (2013) 2013 ACCF/ AHA guideline for the management of ST-elevation myocardial infarction: a report of the American College of Cardiology Foundation/American Heart Association Task Force on practice guidelines. Circulation 127(4):e362-425

5. Chew DP, Scott IA, Cullen L et al (2016) National Heart Foundation of Australia \& Cardiac Society of Australia and New Zealand: Australian clinical guidelines for the management of acute coronary syndromes 2016. Heart Lung Circ 25(9):895-951

6. Ibanez B, James S, Agewall S et al (2018) 2017 ESC Guidelines for the management of acute myocardial infarction in patients presenting with ST-segment elevation: The Task Force for the management of acute myocardial infarction in patients presenting with ST-segment elevation of the European Society of Cardiology (ESC). Eur Heart J 39(2):119-177

7. Task Force JP, Bassand CWH et al (2007) Guidelines for the diagnosis and treatment of non-ST-segment elevation acute coronary syndromes. Eur Heart J 28(13):1598-1660

8. Amsterdam EA, Wenger NK, Brindis RG et al (2014) 2014 AHA/ACC guideline for the management of patients with nonST-elevation acute coronary syndromes: executive summary: a report of the American College of Cardiology/American Heart Association Task Force on Practice Guidelines. Circulation 130(25):2354-2394

9. Elgendy IY, Kumbhani DJ, Mahmoud AN et al (2016) Routine invasive versus selective invasive strategies for Non-ST-elevation acute coronary syndromes: an updated meta-analysis of randomized trials. Catheter Cardiovasc Interv 88(5):765-774

10. Ajani AE, Szto G, Duffy SJ et al (2006) The foundation and launch of the Melbourne Interventional Group: a collaborative interventional cardiology project. Heart Lung Circ 15(1):44-47

11. Peck KY, Andrianopoulos N, Dinh D et al (2021) Role of beta blockers following percutaneous coronary intervention for acute coronary syndrome. Heart 107(9):728-733

12. Kim YH, Her AY, Jeong MH et al (2019) Impact of renin-angiotensin system inhibitors on long-term clinical outcomes in patients with acute myocardial infarction treated with successful percutaneous coronary intervention with drug-eluting stents: comparison between STEMI and NSTEMI. Atherosclerosis 280:166-173

13. Gonzalez-Cambeiro MC, Lopez-Lopez A, Abu-Assi E et al (2016) Mortality benefit of long-term angiotensin-converting 
enzyme inhibitors or angiotensin receptor blockers after successful percutaneous coronary intervention in non-ST elevation acute myocardial infarction. Rev Port Cardiol 35(12):645-653

14. Hara M, Sakata Y, Nakatani D et al (2014) Comparison of 5-year survival after acute myocardial infarction using angiotensin-converting enzyme inhibitor versus angiotensin II receptor blocker. Am J Cardiol 114(1):1-8

15. Pfeffer MA, Braunwald E, Moye LA, The SAVE Investigators et al (1992) Effect of captopril on mortality and morbidity in patients with left ventricular dysfunction after myocardial infarction. Results of the survival and ventricular enlargement trial. N Engl J Med 327(10):669-677

16. Kober L, Torp-Pedersen C, Carlsen JE et al (1995) A clinical trial of the angiotensin-converting-enzyme inhibitor trandolapril in patients with left ventricular dysfunction after myocardial infarction. N Engl J Med 333(25):1670-1676

17. The Acute Infarction Ramipril Efficacy (AIRE) Study Investigators (1993) Effect of ramipril on mortality and morbidity of survivors of acute myocardial infarction with clinical evidence of heart failure. Lancet 342(8875):821-828

18. Heart Outcomes Prevention Evaluation Study Investigators, Yusuf S, Sleight P et al (2000) Effects of an angiotensin-convertingenzyme inhibitor, ramipril, on cardiovascular events in high-risk patients. N Engl J Med 342(3):145-153

19. Fox KM, I.n.v.e.s.t.i.g.a.t.o.r.s. EUROPA (2003) Efficacy of perindopril in reduction of cardiovascular events among patients with stable coronary artery disease: randomised, double-blind, placebo-controlled, multicentre trial (the EUROPA study). Lancet 362(9386):782-788

20. Braunwald E, Domanski MJ, Fowler SE et al (2004) Angiotensinconverting-enzyme inhibition in stable coronary artery disease. $\mathrm{N}$ Engl J Med 351(20):2058-2068

21. Kondo J, Sone T, Tsuboi H et al (2003) Effects of low-dose angiotensin II receptor blocker candesartan on cardiovascular events in patients with coronary artery disease. Am Heart J 146(6):E20

22. Parashar A, Agarwal S, Krishnaswamy A et al (2015) Reninangiotensin system antagonists in patients without left ventricular dysfunction after percutaneous intervention for ST-segment elevation myocardial infarction. Am J Cardiol 116(4):508-514

23. Kim YH, Her AY, Jeong MH et al (2020) ACE inhibitors versus ARBs in patients with NSTEMI with preserved LV systolic function who underwent PCI with new generation drug-eluting stents. Angiology 71(2):139-149

24. Yang JH, Hahn JY, Song YB et al (2014) Angiotensin receptor blocker in patients with ST segment elevation myocardial infarction with preserved left ventricular systolic function: prospective cohort study. BMJ 349:g6650 